\title{
Control of the diffracted response of a metallic wire array with double period: experimental demonstration
}

\author{
D. C. Skigin, ${ }^{1, *}$ J. D. Fowlkes, ${ }^{2}$ N. A. Roberts, ${ }^{3}$ L. B. Scaffardi, ${ }^{4,5}$ D. C. Schinca, ${ }^{4,5}$ and M. Lester ${ }^{6}$ \\ ${ }^{1}$ Grupo de Electromagnetismo Aplicado, Departamento de Física, Facultad de Ciencias Exactas y Naturales, \\ Universidad de Buenos Aires, IFIBA-CONICET, Buenos Aires, Argentina \\ ${ }^{2}$ Center for Nanophase Materials Sciences, Oak Ridge National. Oak Ridge National Laboratory, \\ Oak Ridge, Tennessee 37381-6493, USA \\ ${ }^{3}$ Mechanical and Aerospace Engineering, Utah State University, Logan, Utah 84322, USA \\ ${ }^{4}$ Departamento de Ciencias Básicas, Facultad de Ingeniería, Universidad Nacional de La Plata, Argentina \\ ${ }^{5}$ Centro de Investigaciones Opticas (CIOp), (CONICET La Plata-CIC), La Plata, Argentina \\ ${ }^{6}$ Grupo de Óptica de Sólidos-Elfo, CIFICEN-CONICET, IFAS, and Universidad Nacional del \\ Centro de la Provincia de Buenos Aires, Tandil, Argentina \\ *Corresponding author: dcs@df.uba.ar
}

Received August 4, 2014; accepted August 20, 2014;

posted August 27, 2014 (Doc. ID 217261); published September 29, 2014

In recent papers, it has been theoretically shown that by using dual-period wire gratings, it is possible to control the relative efficiencies of the diffracted orders, regardless of the wires' material, incident polarization and wavelength. In this Letter, we experimentally demonstrate, for the first time, that by appropriately choosing the geometrical parameters of a nanometric periodic structure, it is possible to control the optical response in the visible range. We show examples of nanostructures designed to cancel out or to intensify a particular diffraction order. Such nanostructures allow a broad control over the directionality and the intensity of the diffracted light, which makes them useful for applications such as highly directional optical nanoantennas and photonic multiplexers. (C) 2014 Optical Society of America

OCIS codes: (050.1950) Diffraction gratings; (050.6624) Subwavelength structures; (220.4241) Nanostructure fabrication.

http://dx.doi.org/10.1364/OL.39.005693

Infinite periodic structures with two characteristic periods (dual-period structures) have recently received special attention, because they can be engineered for a specific optical response by controlling geometrical parameters. For instance, a double-period diffraction grating attached to a photonic crystal allows a directional control of the propagating waves, which makes it work like an optical diode [1]. Dual-period dielectric gratings have also been proposed to control the optical response in nanoantennas [2-5] and to introduce significant changes in the reflected and transmitted response of regular periodic structures [ $\underline{6}-\underline{13}]$.

In recent papers, it was shown that both perfectly conducting compound gratings with rectangular grooves and metallic wire compound gratings with circular profiles can be designed to cancel as well as to intensify a given diffraction order [2-4]. This behavior was predicted by a simple model based on the scalar diffraction theory applied to a finite number of slits of subwavelength thickness on an opaque screen. It was corroborated by the modal method for wire structures of finite thickness [2] and for the complementary structure using the integral method based on the second Green's identity [3]].

In this Letter, we compare our theoretical scalar predictions with experimental results in the optical range. These experimental results confirm that nanoscale dualperiod arrays constitute very promising structures that can be useful for many applications and devices, such as optical angular sensing [14], modulators, multiplexers and optical switches [15].

The dual-period nanostructure is formed by metallic wires distributed in groups (sub-arrays) of $J$ nanowires each, as shown in Fig. 1 . A total of $N$ wires are distributed in $M$ subarrays. The structure is periodic (period $D)$ and each sub-array of $J$ nanowires forms a finite grating of period $d$. In Fig. 1 we show a scheme of a structure with $J=5$. The height $h$ and the width $a$ of the wires are also indicated.

Taking advantage of the analogy between slits' and wires' dual-period structures regarding their intensification/cancellation properties (Babinet's principle) [3], we apply the simplified model developed in [2] to predict the electromagnetic response of compound fabricated nanostructures. For this purpose, we consider an opaque screen with slits of width $a$-instead of subwavelength wires-distributed in sub-arrays.

The basic idea of the proposed model is that the response of the dual-period structure can be regarded as a combination of the responses of the periodic structure of period $D$ [16], and that of each sub-array of period $d$. The intensity at a given point $p$ on a screen far from the dual-period slit structure is [2]:

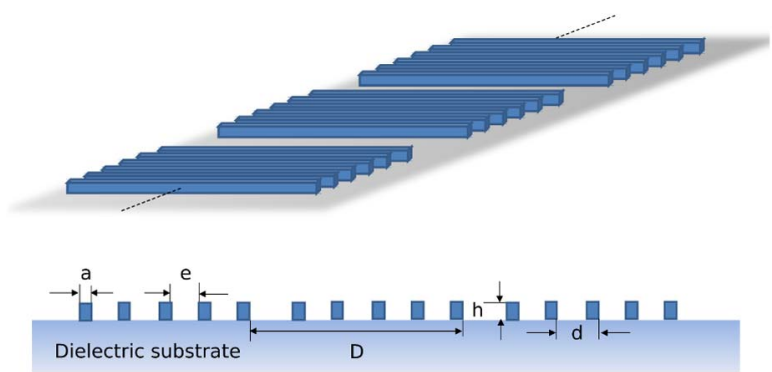

Fig. 1. Scheme of the fabricated dual-period nanostructures. In this case, only three sub-arrays of the whole structure with $J=5$ are shown. 


$$
I_{p}=I_{0}\left(\frac{\sin M \alpha}{\sin \alpha}\right)^{2}\left(\frac{\sin J \gamma}{\sin \gamma}\right)^{2}\left(\frac{\sin \beta}{\beta}\right)^{2},
$$

where $\quad \alpha=(\pi D / \lambda)\left(\sin \theta-\sin \theta_{0}\right), \quad \gamma=(\pi d / \lambda)(\sin \theta-$ $\left.\sin \theta_{0}\right), \quad \beta=(\pi a / \lambda)\left(\sin \theta-\sin \theta_{0}\right), \quad \theta$ and $\theta_{0}$ are the observation and incidence angles, and $a$ is the width of each slit. The first, second and third factors in the right hand side of Eq. (1) can be identified as the interference of the $M$ sub-arrays, the interference of the $J$ wires in each period, and the diffraction of each element, respectively. The interplay between the three terms, especially the $M$ - and $J$-dependent terms, give rise to particular characteristics of the diffraction pattern and can also be exploited to intensify (or to cancel out) a desired diffraction order. The first factor in Eq. (1) yields sharp peaks in very well-defined positions given by the grating equation: $\quad\left(\sin \theta-\sin \theta_{0}\right)=m \lambda / D ; \quad m \in Z$. The $J$ dependent factor has the same form as the first factor, and it can substantially modify the diffraction pattern. Its maxima are given by $\left(\sin \theta-\sin \theta_{0}\right)=p \lambda / d ; p \in Z$ and its minima are found for $\left(\sin \theta-\sin \theta_{0}\right)=$ $(q / J)(\lambda / d) ; q \in Z ; q / J \notin Z$. According to the specific selection of geometrical parameters, one can manipulate the diffracted intensity in particular directions.

For example, when a minimum of the $J$-dependent factor falls in the same angle that a maximum of the $M$-dependent factor, the diffraction order that propagates along this direction is cancelled. This condition is achieved for $(q / J) \times \lambda / d=m \lambda / D$. On the other hand, if both $M$ - and $J$-dependent factors are maximized for certain value of $\theta$, the intensity diffracted in this direction is increased. This occurs for $p \lambda / d=m \lambda / D$. Notice that according to these relationships, the intensification/ cancellation effects are independent of the working wavelength.

In Figs. 2(a) and 2(b), we show the reflected intensity of two dual-period structures given by the scalar model (red line, open circles) as a function of $\sin (\theta)$, and compare this response with that of a simple grating of the same period $D$ (blue line, solid circles) for normal incidence $\left(\theta_{0}=0^{\circ}\right)$. Figure 2(a) corresponds to the case for which all the even orders are cancelled, and the design of Fig. 2(b) was intended to intensify the -3 order. The effect produced by the dual periodicity in the reflected response can be appreciated: whereas all the diffraction orders have similar intensities for the simple grating case, the dual-period structure strongly modifies this response and redistributes the power by almost cancelling the even orders [Fig. 2(a)] or increasing the -3 order [Fig. 2(b)].

Micrographs of the samples fabricated with the geometrical parameters used for Figs. 2(a) and 2(b) (see caption of Fig. 2) are shown in Figs. 3(a) and 3(b). The total area occupied by the array of Fig. 3( $\bar{a})$ is $6.5 \mu \mathrm{m} \times 5.0 \mu \mathrm{m}$ and that of Fig. $3(\mathrm{~b})$ is $10.5 \mu \mathrm{m} \times \overline{5.0 \mu \mathrm{m}}$.

The grating pattern was exposed into a positive tone, electron-sensitive poly(methylmethacrylate) (PMMA) 495 A4 resist supported on an oxide-coated silicon wafer. The $\mathrm{SiO}_{2}$ thickness was $100 \mathrm{~nm}$. The PMMA coating was spin coated onto the $\mathrm{SiO}_{2}$ surface at $4000 \mathrm{rpm}$ for $45 \mathrm{~s}$. The resist coating was prepared for electron exposure with $185^{\circ} \mathrm{C}$ soft bake on a hot plate for $1 \mathrm{~min}$. The electron beam exposure was performed using a JEOL 9300
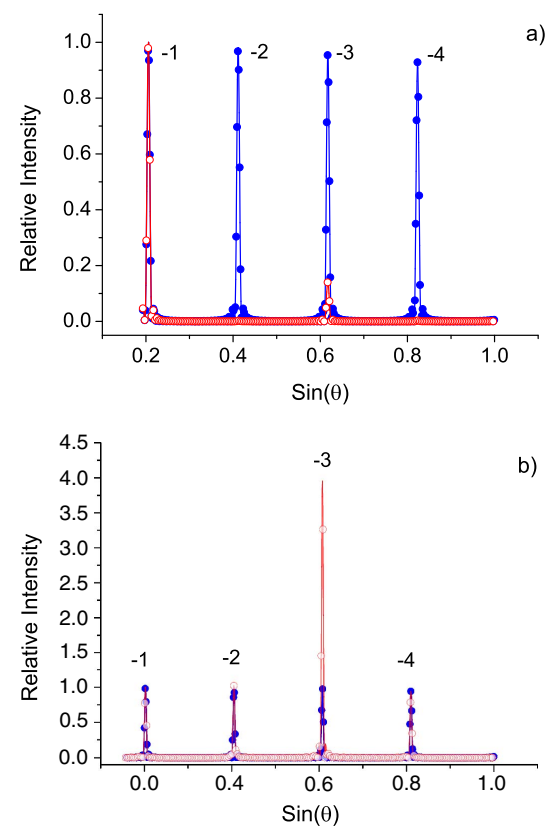

Fig. 2. Reflected intensity obtained by the scalar model (red line, open circles) as a function of $\sin (\theta)$ for two dual-period gratings, in comparison to their counterparts for simple gratings of the same period (blue line, solid circles). The incidence angle is $\theta_{0}=0^{\circ}$, and the intensity is normalized to that of the -1 order in both cases. (a) $J=5, M=25, d=270 \mathrm{~nm}$, $D=2625 \mathrm{~nm}, a=100 \mathrm{~nm}$, and $\lambda=532 \mathrm{~nm}$ (designed to cancel out all even diffraction orders); (b) $J=2, M=39, d=890 \mathrm{~nm}$, $D=2667 \mathrm{~nm}, a=100 \mathrm{~nm}$, and $\lambda=532 \mathrm{~nm}$ (designed to intensify the \pm 3 diffraction orders).

electron beam lithography system. The critical exposure settings consisted of an electron beam accelerating voltage of $100 \mathrm{keV}$ and a primary electron beam current of $2 \mathrm{nA}$. The focused electron probe has a FWHM of $\approx 6 \mathrm{~nm}$. An exposure dose of $1100 \mu \mathrm{C} / \mathrm{cm}^{2}$ was used to fully transfer the grating pattern into the electron sensitive resist. The final development of the PMMA was facilitated by submerging the wafer in a solution of methyl isobutyl ketone (MIBK)/isopropyl alcohol (IPA), with a mixture ratio of $1: 3$ for a total time of $60 \mathrm{~s}$. Upon removing the wafer from the solution, the wafer was rinsed with an IPA stream and subsequently blown dry with a $\mathrm{N}_{2}$ gas flow. PMMA resist was dissolved during this wet development process, in the electron exposed regions, completely to the $\mathrm{SiO}_{2}$ surface. Ni metallization of the exposed grating pattern was performed using an AJA International 200 DC magnetron sputtering system.
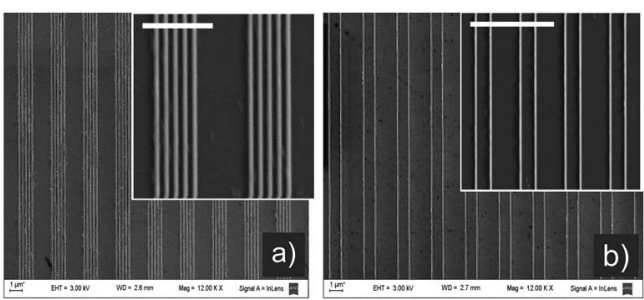

Fig. 3. Detail of the fabricated dual-period nanostructures. Ni wires $25 \mathrm{~nm}$ thick and total length $\approx 5 \mu \mathrm{m}$ were deposited on a $\mathrm{SiO}_{2}$ film. (a) $J=5, M=25, d=270 \mathrm{~nm}$, and $D=2625 \mathrm{~nm}$. Scale bar in the inset is $2 \mu \mathrm{m}$. (b) $J=2, M=39, d=890 \mathrm{~nm}$, and $D=2670 \mathrm{~nm}$. Scale bar in the inset is $4.5 \mu \mathrm{m}$. 


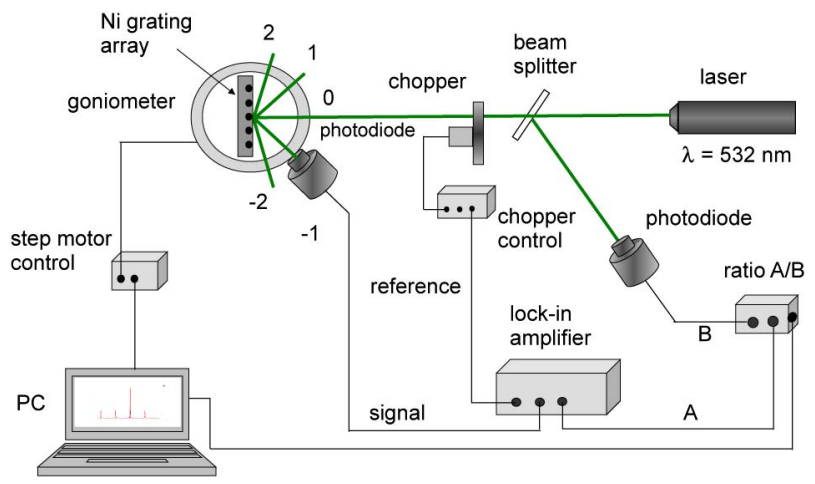

Fig. 4. Schematic of the experimental setup used for angular intensity recording of diffracted orders. Ni grating array is not drawn to scale. Signal photodiode is placed at $1 \mathrm{~m}$ radial distance from the grating.

Sputtering conditions were $30 \mathrm{~W}$, a background chamber pressure of $10 \mathrm{mTorr} \mathrm{Ar}$ and an Ar flow rate of $25 \mathrm{~cm}^{3} / \mathrm{min}$. These conditions lead to a $\mathrm{Ni}$ deposition rate of $4.2 \mathrm{~nm} / \mathrm{min}$. The final grating thickness was $25 \mathrm{~nm} \pm 1 \mathrm{~nm}$ for all samples (see Fig. 3). A final liftoff process consisting of submerging the wafer in a solution of acetone removed the remaining PMMA resist from the unexposed regions of the wafer.

To have a quantitative analysis of the diffracted orders, an angular intensity recording was carried out using a photodiode placed in the moving arm of a goniometer, as sketched in Fig. 4. The grating was mounted on the plate of a $0.05^{\circ}$ angular path step motor driven goniometer. It was home-modified by extending the recording moving arm to a distance of $1 \mathrm{~m}$ to achieve enough spatial resolution for sampling diffracted orders with a sufficient number of points. A low power CW $532 \mathrm{~nm}$ elliptically polarized laser was normally incident on the wire grating, with the major axis of the polarization ellipse is parallel to the nanowires.

The beam was modulated by a chopper at a determined reference frequency for lock-in amplifier (LIA) detection. The diffracted orders were detected by a photodiode attached to the moving arm and its output feeded the LIA. The output of the LIA entered channel A of a ratiometer. Besides, a fraction of the incident beam was taken by a beamsplitter and recorded with a second photodiode that feeds into channel B of the ratiometer to account for possible long-term laser intensity fluctuations. A PC drove the goniometer control and recorded the $\mathrm{DC}$ signal of the $\mathrm{A} / \mathrm{B}$ ratio.

In Fig. $\underline{5}$, we show the optical intensity measured from the arrays of Figs. 3(a) and 3(b), and compare them with their corresponding theoretical predictions made using the scalar approach (Fig. 2). As expressed by Eq. (1), the dual-periodicity of the structure allows new effects that are forbidden for simple or regular gratings. In both cases, a very good agreement can be observed between the experimental and the theoretical results, not only in the angular distribution but also in the relative intensities of the diffraction orders. The main discrepancies between the theoretical and the experimental results can be explained by taking into account that the scalar model does not consider the interaction between the $\mathrm{SiO}_{2}$ substrate and the metallic wires. Besides, the measured

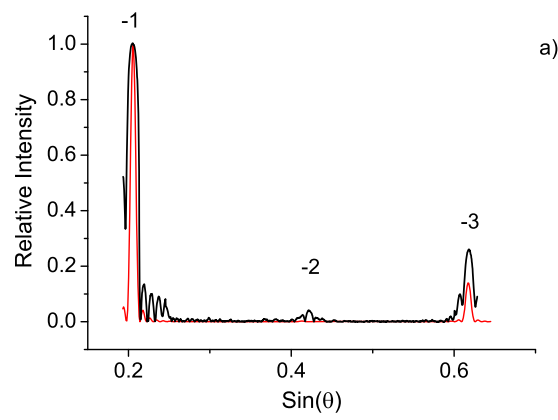

a)

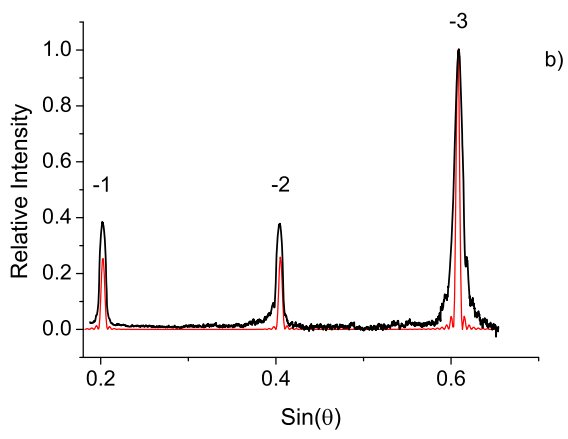

Fig. 5. Experimental reflected intensity (black thick line) as a function of $\sin \theta$ for the dual-period fabricated nanostructures. The measured intensity is compared with the theoretical results obtained by the scalar model (red thin line) for $\lambda=532 \mathrm{~nm}$. (a) The structure parameters are the same as those of Figs. 2(a) and 3(a), for which the -2 order is almost cancelled. In this case, the relative intensity is normalized to that of the -1 order; (b) the structure parameters are the same as those of Figs. 2(b) and 3(b), for which the -3 order is intensified. In this case, the relative intensity is normalized to that of the -3 order.

intensity is expected to be polarization dependent, although expression (1), derived from a scalar model, is independent of the incident polarization mode.

Although the fabricated samples have imperfections, the intensity distribution does not seem to be altered by this, thus exhibiting a high degree of tolerance to fabrication errors. This shows that dual-period nanostructures can be used within nanodevices to control and redirect the electromagnetic intensity. On the other hand, the excellent agreement between theoretical and experimental curves confirms the potential of the simple model to be used as a design tool.

To complete the picture, in Fig. $\underline{6}$, we show photographs of the observed diffraction patters of both nanogratings considered in this work. Figure 6(a) corresponds to the attenuation of the -2 diffraction order and Fig. 6(b) to the intensification of the -3 order. Instead of a simple light spot, order -1 appears in Fig. 6(a) as a diffraction pattern of a rectangular aperture. This pattern corresponds to the whole fabricated nanostructure, since the illuminated area is much larger than the grating. The same feature is observed in the -3 order in Fig. 6(b), in which case this diffraction order is enhanced.

We have provided experimental evidence of the ability of dual-period gratings to distribute the intensity among the diffraction orders according to predesigned requirements. To the best of our knowledge, this is the first time that this phenomenon is experimentally shown within the optical range. The possibility of controlling the intensity distribution by means of a passive device would allow 


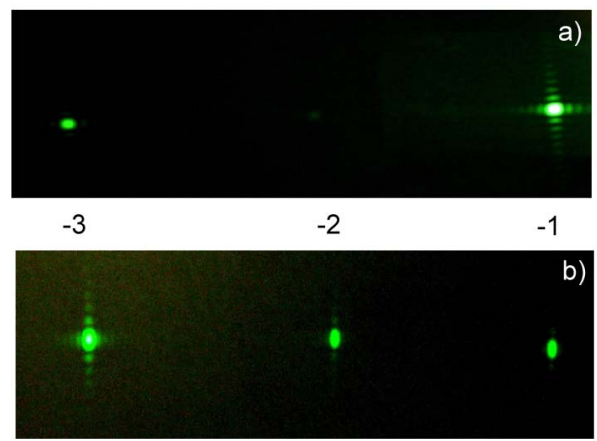

Fig. 6. Photographed images of the diffracted orders corresponding to the spectra shown in Fig. 5. (a) For the structure considered in Fig. 5(a), the cancellation of the -2 order is evident; (b) for the structure considered in Fig. 5(b), a clear intensification of the -3 order is observed.

many applications in nanotechnology, such as optical multiplexers and photonic logic gates.

ML thanks Dr. Eduardo Caselli and Dr. Javier Diez for their collaboration and comments. ML gratefully acknowledges support from CONICET (PIP 0145) and UNICEN; DS acknowledges support from CONICET (PIP 112-200801-01880) and UBACyT (20020100100533); LBS and DCS acknowledge support fron CONICET (PIP 0394) and grant 11/I151 from Facultad de Ingeniera, UNLP. A portion of this research was conducted at the Center for Nanophase Materials Sciences, which is sponsored at Oak Ridge National Laboratory by the Scientific User Facilities Division, Office of Basic Energy Sciences, U. S. Department of Energy.

\section{References}

1. A. Serebryannikov, E. Colak, and A. Cakmak, Opt. Lett. 37, 4844 (2012).

2. D. C. Skigin and R. A. Depine, Appl. Opt. 46, 1385 (2007).

3. M. Lester, D. C. Skigin, and R. A. Depine, Appl. Opt. 47, 1711 (2008).

4. M. Lester, D. C. Skigin, and R. A. Depine, J. Opt. A Pure Appl. Opt. 11, 045705 (2009).

5. I. M. Mandel, E. Lansey, J. N. Gollub, C. H. Sarantos, R. Akhmechet, A. B. Golovin, and D. T. Crouse, Appl. Phys. Lett. 103, 251116 (2013).

6. D. C. Skigin and R. A. Depine, Phys. Rev. Lett. 95, 21, 217402 (2005).

7. D. C. Skigin and R. A. Depine, Opt. Commun. 262, 270 (2006).

8. D. C. Skigin and R. A. Depine, Phys. Rev. E 74, 046606 (2006).

9. A. P. Hibbins, I. R. Hooper, M. J. Lockyear, and J. R. Sambles, Phys. Rev. Lett. 96, 257402 (2006).

10. M. Navarro-Cía, D. C. Skigin, M. Beruete, and M. Sorolla, Appl. Phys. Lett. 94, 091107 (2009).

11. I. Bendoym, A. B. Golovin, and D. T. Crouse, Opt. Express 20, 22830 (2012).

12. D. Crouse, E. Jaquay, A. Maikal, and A. P. Hibbins, Phys. Rev. B 77, 195437 (2008).

13. I. M. Mandel, A. B. Golovin, and D. T. Crouse, Phys. Rev. A 87, 053833 (2013).

14. K.-X. Sun, S. Buchman, and R. Byer, J. Phys. 32, 167 (2006).

15. J. He, R. A. Norwood, M. Brandt-Pearce, I. B. Djordjevic, M. Cvijetic, S. Subramaniam, R. Himmelhuber, C. Reynolds, P. Blanche, B. Lynn, and N. Peyghambarian, Comput. Electr. Eng. 40, 216 (2014).

16. M. Born and E. Wolf, Principles of Optics, 4th ed. (Pergamon, 1970). 